\title{
Atributos de la personalidad de marca socialmente responsable
}

\author{
Attributes of a socially responsible brand personality
}

Javier Mayorga Gordillo. Universidad Autónoma de Occidente. Colombia.

jmayorga@uao.edu.co

[CV]

Elena Añaños Carrasco. Universidad Autónoma de Barcelona. España.

elena.ananos@uab.cat

$[\underline{\mathrm{CV}}]$

Cómo citar este artículo / Referencia normalizada

Mayorga Gordillo, J. y Añaños Carrasco, E. (2020). Atributos de la personalidad de marca socialmente responsable. Revista Latina de Comunicación Social, 75, 97-120.

https://www.doi.org/10.4185/RLCS-2020-1418

\section{RESUMEN}

En el contexto actual donde los gobiernos, las empresas y en general la sociedad están en búsqueda de poner fin a la pobreza, proteger el planeta y garantizar que las personas gocen de paz y prosperidad, las acciones de tipo social gestionadas por las organizaciones se han convertido en un elemento que puede influir en la percepción positiva que tienen los públicos de las mismas. Es por este motivo que diversos estudios (Becker-Olsen, Cudmore, y Hill, 2006; Du, Bhattacharya, y Sen, 2007; Hildebrand, Demotta, Sen, y Valenzuela, 2017; Hoeffler y Keller, 2002; Madden, Roth, y Dillon, 2012; Manzano, Simó, y Pérez, 2013; Rivera, Bigne, y Curras-Perez, 2016; Sen y Bhattacharya, 2001, entre otros) se han centrado en analizar los efectos de las acciones de responsabilidad social corporativa (RSC), llegando a concluir que pueden ser un factor que modifica positivamente las percepciones de los consumidores sobre una empresa en su conjunto y de su rol en la sociedad, logrando afectar significativamente la solidez y el valor de una marca. En esta línea, este trabajo analiza los atributos comunicacionales que son percibidos como rasgos determinantes de una personalidad de marca socialmente responsable. Dichos rasgos son valorados por 385 sujetos de diferentes nacionalidades con el objetivo de identificar las asociaciones y las agrupaciones de atributos que permiten la construcción de la personalidad de las marcas socialmente responsables. Los resultados obtenidos muestran la existencia de diversos factores que influyen en la valoración de los atributos estudiados y permiten concluir que existe una estructura relacional de atributos que pueden conformar una nueva dimensión de las marcas dotándolas de una personalidad socialmente responsable que complemente y engrandezca los propios valores de la organización en su conjunto.

PALABRAS CLAVE: branding; marca socialmente responsable; responsabilidad social; personalidad de marca; gestión de marca; comunicación corporativa. 


\section{ABSTRACT}

In the current context where governments, companies and in general, the society are aiming to end poverty, protect the planet and guarantee that people live in peace and prosperity, the social actions undertaken by organisations have turned into an element that can influence the positive perception evoked in the public. Therefore, different studies (Becker-Olsen, Cudmore and Hill, 2006; Du, Bhattacharya and Sen, 2007; Hildebrand, Demotta, Sen and Valenzuela, 2017; Hoeffler and Keller, 2002; Madden, Roth and Dillon, 2012; Manzano, Simó and Pérez, 2013; Rivera, Bigne and Curras-Perez, 2016; Sen and Bhattacharya, 2001, among others) have focused on analysing the effects of corporate social responsibility (CSR), concluding that they might be a factor that positively modifies the perceptions of consumers about a company and its role in the society, significantly impacting the strength and value of a brand. In this line, this study analyses the communication attributes perceived as determinant traits of a socially responsible brand personality. These traits are assessed by 385 individuals from different nationalities in order to identify the associations and clusters of attributes that allow the construction of a socially responsible brand personality. The results obtained show the existence of different factors that influence the assessment of studied attributes and allow to conclude that there is a relational structure of attributes that can constitute a new dimension of brands, endowing them of a socially responsible personality that complements and enhances the values of the organisation altogether.

KEYWORDS: branding; socially responsible brand; social responsability; brand personality; brand management; corporate communication.

\section{CONTENIDO}

1. Introducción. 1.1. Marco Contextual. 1.1.1. Contexto actual de la sociedad: La era del Desarrollo Sostenible. 1.1.2. La Marca como elemento de competitividad corporativa. 1.1.3. La Marca como elemento relacional de la identidad corporativa. 1.1.4. La personalidad de la marca como elemento diferenciador de la imagen de la marca. 2. Método. 2.1. Instrumentos de recogida de información. 2.2. Población y muestra. 2.3. Variables y Tipo de Diseño. 2.4. Procedimiento. 3. Resultados. 3.1. Descripción de los sujetos participantes. 3.2. Resultados del análisis de los atributos que componen la dimensión de la personalidad de marca socialmente responsable. 4. Discusión y conclusiones. 5. Referencias bibliográficas.

Traducido por Yuhanny Henares (traductora académica, Universitat de Barcelona).

\section{Introducción}

Siendo la marca un elemento comunicacional visible de las organizaciones, y atendiendo al contexto actual de la sociedad, se reclama un comportamiento responsable por parte de las mismas y se hace necesario incorporar a los modelos de gestión de la marca, herramientas que contribuyan con su rol de agente de cambio social. Por tal motivo, este trabajo esboza la nueva concepción de la marca tomando en cuenta los conceptos de responsabilidad social y sostenibilidad los cuales permitan identificar los atributos y rasgos de la personalidad de la marca que contribuyan a su gestión y a su vez relacionarla con sus audiencias de una forma acorde al comportamiento empresarial que sus productores profesan en su día a día corporativo.

Con este trabajo se pretende analizar las características que reflejan una personalidad con una marcada sensibilidad social, y seleccionar las más pertinentes y relevantes para describirla a la luz del análisis 
estadístico de las valoraciones que hacen los sujetos participantes en la investigación, complementando así el modelo de atributos de la personalidad de la marca realizado por Aaker (1997)y permitiendo enmarcar dicho modelo en el contexto actual en el cual las marcas interactúan con la sociedad y los individuos y también con el medioambiente.

\subsection{Marco Contextual}

La sociedad se encuentra en un proceso de cambio que vislumbra una evolución en relación a su contexto ambiental, económico, político, tecnológico y en su organización social como tal. Diversos autores han planteado que en medio de este proceso de cambio, ha aparecido un nuevo tipo de consumidor, consciente de sus actos de compra, con unos valores diferentes y preocupado por sus acciones en el mercado (Crane y Matten, 2004; Hendarwan, 2002; Rochefort, 1995; Strong, 1996) citados por (Dueñas Ocampo, Perdomo-Ortiz, y Villa Castaño, 2014). Este hecho ha llevado a que las organizaciones, tratando de interpretar dicha situación de trasformación de la sociedad y principalmente de los consumidores, busquen nuevas formas de establecer relaciones con sus audiencias desde una perspectiva más comprometida y responsable, es decir mucho más sostenible.

Desde este nuevo punto de vista del consumidor responsable, aumenta el interés por la procedencia de los productos y su proceso productivo y manufacturero, valorando la no violación de las normas globales de trabajo justo y el cumplimiento de los derechos humanos, aumentándose la preocupación por el incremento de la industria militar, la corrupción estatal y el maltrato animal, entre otros muchos aspectos que afectan la decisión de adquisición y uso de algún bien o servicio (Newholm y Shaw, 2007).

Así, el consumidor actual es cada vez más consciente que las decisiones tomadas en el pasado en cuestiones políticas, ambientales, sociales y económicas han ido generando cambios categóricos en la calidad de vida, la equidad, el medio ambiente y el bienestar; este hecho genera la necesidad de replantear no solo los modelos económicos y políticos que rigen la mayoría de estados, sino también aquellos relacionados con la producción y el consumo humano.

\subsubsection{Contexto actual de la sociedad: la era del Desarrollo Sostenible}

En base a lo expuesto anteriormente, se puede observar que las nuevas generaciones son críticas con el modelo de mercado actual y están muy preocupadas porque el crecimiento del mercado libre propicie, entre otras cosas, la contaminación y exacerbe la desigualdad de ingresos. Pero aunque pareciera paradójico, entienden que el modelo de la sociedad actual cuenta con adelantos tecnológicos y comunicacionales que lo pueden favorecer (Kahn, 2015). Así, una gran parte de la sociedad quiere tácitamente ser próspera y aspira a que la pobreza mundial disminuya para que las futuras generaciones puedan vivir mejor; otros se preocupan por los desafíos ambientales, el cambio climático, la contaminación del aire, la protección de la biodiversidad y el agotamiento de los recursos naturales claves para el futuro de la humanidad y su ecosistema; pero muy pocos comprenden la visión liberal del crecimiento del libre mercado para reducir la pobreza global (Kahn, 2015).

Es por estas y otras razones que los gobiernos y grandes líderes políticos y de opinión, liderados por Naciones Unidas, presentan en 2015 el Plan 2030 para el Desarrollo Sostenible Mundial y junto con los Objetivos de Desarrollo Sostenible (ODS), cuya agenda guía los gobiernos del mundo. 
Según Kahn (2015) el Desarrollo Sostenible se puede entender como una búsqueda intelectual que intenta dar sentido a las interacciones entre la economía mundial, la sociedad global y el entorno físico de la Tierra. Este autor lo plantea desde dos enfoques: el positivo y el normativo. El positivo parte de una mirada académica en la que se pueden analizar las interacciones y fricciones entre el sistema económico-social y el medioambiental; es decir, se centra en la interacción del individuo y sus colectivos con el medio natural que los cobija, permitiendo interpretar qué consecuencias tienen dichas relaciones en las que coexisten; el enfoque normativo permite obtener una perspectiva del diseño de políticas públicas y de su relación con los diferentes actores sociales.

Desde los inicios de este siglo, diversos autores, líderes y sociedad civil, entre otros, han intentado explicar y proponer modelos para lograr un desarrollo socioeconómico ambiental respaldado en un sistema económico que promueva la equidad y el cuidado del medio ambiente. En este sentido, Sachs y Vilà (2015) plantean que esta forma de entender el nuevo mundo desde una visión sostenible busca que el crecimiento y progreso se base en la eliminación de la pobreza extrema y en el planteamiento de políticas dirigidas a reforzar las comunidades, para lograr incrementar así la confianza social además del cuidado y la protección del medio ambiente del ataque sistémico y sistemático que ha provocado el ser humano durante siglos. Según estos autores, el desarrollo sostenible necesita de una mirada holística puesto que la sociedad persigue simultáneamente objetivos sociales, económicos y ambientales.

Todo ello justifica que la Agenda 2030 pretenda ser un plan que coloque como eje central la dignidad e igualdad del individuo. Es esta una apuesta ambiciosa y visionaria y requiere de la participación de diversas esferas de la sociedad (representantes de los gobiernos, la sociedad civil, el ámbito académico, el sector privado, etc.) que la implementen como una herramienta para la creación de sociedades inclusivas y justas, al servicio tanto de las personas de hoy como de las futuras generaciones (CEPAL, 2018).

"El sector empresarial debe entender que actualmente y en un futuro, es necesario transformar el paradigma de desarrollo dominante en uno que nos lleve por la vía del desarrollo sostenible, inclusivo y que tenga una visión de largo plazo" (CEPAL, 2018), entendiendo que para ello se requiere promover el bienestar de todos los individuos, más allá del simple cumplimiento del estado de bienestar social, del que gozan algunas naciones, pero que mayoritariamente el mundo no ha logrado conseguir para sus ciudadanos.

Según Sachs y Vilà (2015), para lograr los objetivos de esta agenda es necesario que exista una buena gobernanza que promueva un sano equilibrio entre el estado, la sociedad civil y el mercado de la economía (RAE, 2018). Para conseguirlo, estos autores afirman que las empresas tienen un rol protagónico y que la buena gobernanza no es una actividad exclusiva de los gobiernos, sino que también las grandes empresas transnacionales son actores con un gran poder político, económico y social. Así, el bienestar de la humanidad, se verá afectado positivamente si estas grandes empresas cumplen la ley y respetan y cuidan el ambiente, contribuyendo así a la mejora continua de las comunidades en las que están presentes, es decir son agentes de cambio comprometidos con el progreso de la sociedad.

Así pues, las empresas deben ser no sólo medios de producción sino agentes de cambio que buscan maximizar beneficios. Anteriormente esos beneficios eran básicamente de tipo económico, pero ahora la sociedad reclama que tengan la misma importancia los beneficios sociales y ambientales; es por ello que las empresas deben trabajar por la transformación y la mejora de sus entornos locales desde una 
perspectiva global, tomando como base al individuo, la sociedad y el ecosistema. No se puede olvidar que las empresas son el centro del tejido productivo y económico de las naciones, por lo que deben pensar en el bien común para de esa forma construir y modificar la sociedad actual y futura. Bajo esta perspectiva, Kotler, Kartajaya y Setiawan (2010) consideran que se está viviendo una era centrada en los valores en la que las organizaciones no ven a los individuos como unos simples consumidores, sino que los conciben como seres humanos con inteligencia, corazón y espíritu, por lo que se entiende que, en su formulación estratégica, las empresas de hoy deben incluir aspectos como la justicia social, económica y medioambiental, lo que las diferenciará en el momento de la toma de decisión de compra, ya que el consumidor actual no busca sólo una mera satisfacción funcional, sino también una satisfacción más de tipo emocional. Así, las empresas que sustentan su gestión en los valores podrán ofrecer respuestas y esperanzas a quienes se enfrentan a los problemas actuales de la sociedad (pobreza, desigualdad, inequidad, destrucción medioambiental, entre otros) y se relacionarán con los consumidores en un nivel superior (Kotler et al., 2010).

\subsubsection{La Marca como elemento de competitividad corporativa}

La marca es actualmente una prioridad gerencial debido a la creciente tendencia que existe de que sea uno de los activos intangibles más valiosos que tengan las empresas. Las marcas cumplen varias funciones fundamentales en el proceso de relación y promoción de las empresas y de sus bienes o servicios. Para los clientes las marcas pueden simplificar la elección, prometer un nivel de calidad particular, reducir el riesgo y generar confianza; de este modo, uno de los aspectos relevantes de la marca lo forman sus intangibles, es decir, aquellos aspectos relacionados con la imagen de la marca que no involucran los atributos o beneficios físicos concretos del producto (Keller y Lehman, 2006).

En base a la relevancia de la marca en la actualidad, la definimos como una creación conjunta entre las audiencias y los fabricantes, de la cual se genera un conjunto de elementos y signos comunicaciones de tipo racional y emocional que al relacionarse entre sí producen un significante, el cual permite no solo identificar a un producto o servicios, sino también identificarlo y diferenciarlo dentro del contexto social en el que convive y del mercado en el que interactúa (Mayorga, 2017). Así, la marca es mucho más que un nombre, término, diseño, símbolo o cualquier otra característica que (solo) identifique el bien o servicio de un vendedor a diferencia de los de otros vendedores (AMA, 2015). Además, según los estándares ISO (2018), la marca pretende crear en la mente de los diferentes públicos, imágenes y asociaciones distintivas, generando así beneficios para las organizaciones y tiene como función, el establecimiento de una identidad diferenciadora de la propia organización o producto. Para ello debe comunicar los beneficios únicos de dicha entidad, considerando que éstos pueden ser tanto funcionales como emocionales o sociales.

Diversos investigadores han desarrollado trabajos en la búsqueda de mejores formas de enfrentar los desafíos de las marcas en la actualidad. Desde finales del siglo XX, se han propuesto diferentes conceptos que han permitido entender la importancia que las marcas tienen en el proceso de relación con sus audiencias; la mayoría convergen en la idea que el propósito principal de una marca es aumentar el valor comercial del producto/servicio que representa y el de la firma productora, reducir el riesgo económico y extender la existencia sostenible del productor. Desde una visión contemporánea, la marca contribuye a atraer a los clientes y a aumentar los ingresos a través de un incremento en las primas de precios y/o volúmenes, además de contribuir en el incremento de la fidelización (ISO, 2018). 
Así, más allá de que las marcas pueden reducir costos, crear una ventaja competitiva e impactar en los ingresos y la rentabilidad, también pueden influir en el valor corporativo: para ello es necesario que el valor de la marca no se centre únicamente en el rendimiento financiero que puede aportar a las organizaciones, sino que se deben desarrollar modelos de gestión de la marca en los que sea más relevante el efecto de las actividades de marketing que afectan al comportamiento del mercado, generando así el valor en la marca como bien de transacción, bien emocional y como activo intangible de las compañías (Faircloth, Capella y Alford, 2001).

Profesionales e investigadores afirman que en la actualidad se pretende construir marcas fuertes, es decir, se busca incrementar el nivel de afectación positiva de estas sobre los clientes o diferentes stakeholders, utilizando para ello estrategias que persiguen la solidez legal de la marca y el fortalecimiento de la percepción de los consumidores. Es por eso que, para poder conocer el valor de una marca, es necesario seleccionar los elementos, dimensiones e indicadores que le son más apropiados y relevantes (ISO, 2018). En este sentido, una marca debe perseguir el logro de un posicionamiento clave, establecer asociaciones en la mente de los clientes y desarrollar otros componentes importantes que la diferencien de las demás y le permitan alcanzar una superioridad competitiva Keller y Lehman (2006). Desde una perspectiva relacional, se entiende que los consumidores generan asociaciones y vínculos fuertes con las marcas de una forma multifacética, es decir, no se puede determinar que existe un único elemento que produzca una relación duradera entre marca y audiencia. Según Fournier (citado en Keller y Lehman, 2006) existen seis dimensiones, más allá de la lealtad o el compromiso, a lo largo de las cuales las relaciones entre la marca y el consumidor varían: la conexión de auto-concepto, el compromiso o apego nostálgico, la interdependencia del comportamiento, el amor/pasión que desencadena, la intimidad y la propia marca calidad del socio.

Según Faircloth et al. (2001), la respuesta diferencial es el resultado de un efecto mental de conocimiento de la marca por parte de los consumidores, que se sustenta en su imagen y en su reconocimiento. Estos autores consideran que la imagen de marca la forman las creencias y cogniciones perceptivas que las audiencias tienen sobre los atributos, beneficios y las asociaciones de la actitud de la marca. Es por ello que los directivos de las empresas deberán poner más atención en generar la confianza de sus consumidores, ya que hoy en día los individuos confian más los unos en los otros que en las empresas (Kotler et al., 2010). Según estos autores las empresas deben acogerse al nuevo sistema de confianza del consumidor, en el que su comunidad se erige como agente de cambio, creando sus propios productos y experiencias en colaboración con otros individuos que la conforman; por lo tanto las organizaciones que buscan tener éxito dentro de este sistema deben entender que los consumidores aprecian cada vez más la colaboración en el proceso de creación y desarrollo de la personalidad de una marca.

\subsubsection{La Marca como elemento relacional de la identidad corporativa}

Inmersos en la era del Desarrollo Sostenible, entendemos que el valor de una marca está fuertemente vinculado a la imagen, coherencia y consistencia que se tenga del comportamiento de la organización en relación a los productos y/o servicios, y que se soporta en el reconocimiento de su identidad corporativa. En este contexto, se hace imperativo, conocer el efecto de las acciones que una compañía desarrolla en pro de la mejora de la sociedad, de sus integrantes y del medio ambiente, pero no solo desde el valor financiero, sino también desde la perspectiva relacional, es decir, en base a la forma con la que los 
individuos interpretan dichas acciones como un atributo dominante de la marca que la identifican y la diferencian de las demás.

Las marcas son propensas a generar asociaciones comunes a sus atributos, beneficios y valores (Keller y Lehman, 2006). Cada vez más, estudios empíricos concluyen que los consumidores establecen relaciones más duraderas y rentables con las marcas que manifiestan claramente su carácter social, basado en aspectos como el trato de sus empleados, el impacto sobre el medio ambiente y su aportación a la sociedad. Así, la marca implica más que una promesa; es un vínculo, una relación evolutiva basada en percepciones y experiencias que el cliente experimenta cada vez que se conecta con ella, además de generar beneficios funcionales, de auto expresión y sociales (Casanoves, 2017).

De lo expuesto se deriva la necesidad de que los directivos de las organizaciones entiendan que en un mercado en el que, a la hora de elegir entre empresas, marcas y productos, cada vez es más importante la valoración de las aportaciones a la sostenibilidad, es necesario desarrollar atributos de la propia marca que permitan a las organizaciones generar vínculos cargados de conciencia social y ambiental, lo cual debe ser no solo un ejercicio comunicacional, sino también estratégico, cultural y comportamental. Por ello, las organizaciones deben conservar un equilibrio entre el cumplimiento de las normas, tanto legales como morales, y las actuaciones sobre las personas y el cuidado del medio ambiente, no solo porque todo ello contribuirá con una mejor imagen y valor de la marca, sino porque en un futuro no muy lejano, las empresas que no apunten a este tipo de gestión orientada a la sostenibilidad serán excluidas por el propio mercado.

Cuando una empresa desarrolla estrategias coherentes o nuevos productos y servicios innovadores basados en el concepto de sostenibilidad, a base de materiales eficientes y que se sustentan en un proceso de producción más limpia, permite que los consumidores la sitúen una posición preferencial en el momento de la toma de la decisión de compra, puesto que hoy en día éstos están eligiendo las marcas más responsables. En este contexto de sostenibilidad es necesario que los gestores de la marca entiendan que un elemento fundamental de las marcas del hoy y del mañana se debe centrar en la integridad es decir, en el cumplimiento de lo ofrecido a partir de su posicionamiento y diferenciación, pero también debe mostrar credibilidad cumpliendo con las promesas de valor y estableciendo una relación basada en la confianza.

Uno de los cimientos más importantes de la era del desarrollo sostenible, es la gestión empresarial soportada en valores. Cada vez más, las compañías han adoptado un modelo estratégico basado en la declaración formal de valores corporativos y los directivos actualmente tienden a identificar el comportamiento ético, la honestidad, la integridad y las preocupaciones sociales como ejes centrales de su gestión. Diversas organizaciones manifiestan hoy en día estos valores, permitiendo que los públicos identifiquen sus prioridades corporativas y criterios de gestión en pro de la construcción de una relación coherente y estable con dichas audiencias. Actualmente las compañías no deben basar su gestión únicamente en la rentabilidad y el retorno de la inversión, sino que también deben procurar alcanzar la sostenibilidad del negocio sin afectar la sostenibilidad del entorno.

Casanoves (2017, p. 53) afirma que “(...) la marca es el fiel reflejo de pensamiento, sentimiento y actos del consumidor, y busca conseguir potente conexión emocional con el mismo, ya que este es, sin duda, el eslabón fundamental de la cadena de valor de marca.”; así, si el consumidor está reconstruyendo su estructura de principios, creencias y valores, dándole más importancia a la sostenibilidad, las 
organizaciones deben establecer formas de relación con la sociedad que permitan construir comunidades basadas en esta nueva estructura de valores sociales.

Hoeffler y Keller (2002) ya plantearon la personalidad de la marca como elemento de relación que apela a algunos rasgos distintivos de la personalidad de los consumidores para establecer vínculos fuertes con la misma, siendo uno de ellos la sinceridad, rasgo que ya fue planteado en el modelo de Aaker (1997); además, consideran que es posible que existan otros rasgos de dicho modelo que se ajusten a la personalidad de la marca cuyos contenidos permitan generar lazos entre la marca y la audiencia basados en aspectos sociales y ambientales, de forma que una gestión empresarial sustentada en el interés colectivo del bienestar, permitirá generar valor en las marcas ya que contribuye a crear conciencia de la misma, mejorar su imagen de marca, establecer credibilidad de marca, evocar sentimientos de marca y a crear un sentido de comunidad de la marca que aumenta su compromiso.

\subsubsection{La personalidad de la marca como elemento diferenciador de la imagen de la marca}

Según Kotler et al. (2010), para que se establezca una relación duradera con su público, es necesario que las marcas desarrollen un $\mathrm{ADN}$ autentico que permita la verdadera definición de la marca y la diferencie de las demás. Estos autores consideran que la personalidad de la marca es el medio para lograr emprender la construcción de una identidad y una imagen congruente con este modelo de relacionamiento y diferenciación empresarial. En este sentido las empresas deben buscar la autenticidad y cumplir con la experiencia y las expectativas que ofrecen; su único objetivo no puede ser parecer reales en su publicidad, puesto que, si esto es así, perderán su credibilidad. Así, la personalidad de la marca se convierte en el punto de partida de la gestión de marca como elemento relacional en la era del Desarrollo Sostenible.

Aaker (1997) fue la primera autora que planteó un modelo que permite no solo identificar las dimensiones y atributos de la marca percibidos por las audiencias, sino que además contribuye a la gestión integral de la misma, permitiendo moldearla de una forma tal que los públicos la interpreten y se relacionen con ella de la manera más cercana a lo previsto por la compañía. Esta autora propone un modelo conceptual de asociaciones de la marca formado por los atributos de marca, sus beneficios y las actitudes de marca. Los atributos de una marca son el nivel de asociación más objetivo o concreto y son perceptivos en el sentido que corresponden a lo que el consumidor percibe sobre el atributo y determinan su importancia o esencia (Faircloth et al., 2001).

Faircloth et al. (2001) consideran que las audiencias forman la imagen a través de la suma de todos los estímulos generados por la marca, y afirma que la imagen de ésta es el resultado de los procesos de decodificación, extracción e interpretación por parte de los consumidores. A su vez, la imagen de marca que elabora el consumidor es un resultado de diversas acciones acumuladas que la empresa ha llevado a cabo como parte de su mezcla de marketing, por lo que según una imagen de única, sólida y favorable, permitiendo que ella y su significado se encuentren estratégicamente diferenciados y posicionados en la mente de los consumidores.

Dado que se aprecia la importancia de las asociaciones mentales por parte de los consumidores, en las que se construye el posicionamiento, el recuerdo y el compromiso del individuo con la marca y que por ende constituyen el sustento del posterior valor de misma, es necesario que los productores definan con claridad qué atributos y rasgos contribuyen a su definición con el objetivo que sus audiencias 
reconozcan su alto compromiso social. Esto crea la necesidad de identificar una dimensión de la personalidad de la marca de tipo socialmente responsable, la cual contribuya a la gestión estratégica de la misma y permita establecer una conexión emocional entre consumidor y marca. En este sentido, consideramos muy importante analizar los posibles atributos comunicacionales que se encuentran implícitos en la construcción de una personalidad de la marca que la hagan socialmente responsable.

Dueñas Ocampo et al. (2014) abordan el tema de la personalidad responsable, desde una perspectiva evolutiva del concepto de consumo responsable y consideran que es un concepto que aparece principalmente en la década de los años 50, simultáneamente con la formalización de la responsabilidad social corporativa, como destacan Cochran (2007) y Wartick y Cochran (1985). Los mencionados autores, hacen referencia a los trabajos de Berkowitz y Daniels (1964), Gough, McClosky y Meehl (1952) y Harris (1957) Berkowitz y Lutterman (1968), quienes principalmente parten de las transformaciones sociales y del desarrollo político, particularmente en Estados Unidos, lo que los llevó a determinar las características o el perfil de las personas socialmente responsables y la relación entre su responsabilidad y su comportamiento frente a los diferentes actores sociales.

Hacia los años 70 los investigadores centraron sus investigaciones en el estudio de los consumidores socialmente responsables y los elementos que los caracterizan. Según Dueñas Ocampo et al. (2014), una de las aportaciones más relevantes de esta década fue la de Anderson y Cunningham (1972) quienes afirmaron que la personalidad responsable no implica directamente una acción directa de tipo responsable, es decir no conlleva un consumo socialmente responsable asociado. Estos autores consideran que algunas características demográficas pueden ser asociadas con unas puntuaciones más altas en la escala de responsabilidad social, pero que una personalidad responsable no compromete acciones, por parte del consumidor, que reflejen su comportamiento responsable; hecho por el cual se deben replantear las investigaciones en esta temática y concentrarse, principalmente, en las actitudes del consumidor, para de esa forma poder comprender las motivaciones que se generan en relación también a sus acciones.

A finales de la década de los 90, las investigaciones sobre la personalidad de una marca y su relación con los consumidores, derivan en la formulación de una escala de personalidad de marca, basada en los hallazgos propuestos desde la psicología. Es así como Aaker (1997) plantea un modelo de personalidad de la marca basado en 5 dimensiones, pero en el cual no se detectan explícitamente unos atributos que permitan describir una personalidad socialmente marcada; estas dimensiones son: sinceridad, emocionante, competencia, sofisticación y robustez. Diversos trabajos se han llevado a cabo con posterioridad a la propuesta de Aaker (Chu y Sung, 2011; Cuevas Moreno, 2011; Freling, Crosno, y Henard, 2011; Goñi, Torres, y Aguilera, 2013; Kim, Shim, y Dinnie, 2013; Madrigal y Boush, 2008) entre otros, en los que se intenta reformular su modelo inicial, partiendo de observaciones sobre la complejidad que surge a la hora de generalizar los rasgos planteados por la autora a todos los contextos mundiales. Este hecho ha llevado a la definición e investigación de nuevos atributos y, en algunos casos, a la definición de nuevas dimensiones que conformen la personalidad de una marca. Así por ejemplo, en el contexto indio, los trabajos de Ahmad y Thyagaraj (2017) evidencian la presencia de una nueva dimensión de la personalidad de la marca propuesta por Aaker que consideran identifica mejor a esta sociedad; los autores denominan a esta dimensión Integridad y la relacionan con las características culturales de dicho contexto.

Por otro lado Mayorga (2017) propone la adición de una sexta dimensión al modelo de Aaker, centrada en los rasgos de una personalidad con un marcado interés social. El autor denomina dicha dimensión 
como Sensibilidad, y estima que, al transferir esta virtud humana al contexto de las marcas, éstas pueden ser más sensibles en el momento de relacionarse con sus audiencias, permitiéndoles no ser indiferentes a las problemáticas sociales del contexto donde co-habitan con sus públicos. Esto permite motivar al fabricante a convertirse en un agente de cambio social. Esta virtud de la personalidad de la marca es necesaria para la construcción de relaciones entre las marcas y sus consumidores, ya que genera vínculos especiales entre ellos y contribuye al posicionamiento y la reputación de la marca dentro de sus públicos.

Estos aportes teóricos permiten moldear una relación entre empresa y audiencias sustentada en la sensibilidad de la personalidad de la marca que se encuentra encaminada a posicionar a la marca como elemento estratégico relacional en la era del Desarrollo Sostenible. Desde esta perspectiva, consideramos que es necesario analizar los atributos comunicacionales que formen parte de la definición y la construcción de una personalidad de marca socialmente responsable, entendiéndolos como elementos relacionales de carácter estratégico para las marcas y sus fabricantes.

Es por ello que este trabajo tiene como objetivo seleccionar y analizar las características o atributos que definen y describen la personalidad de una marca socialmente responsable y generar un conjunto de rasgos que esté conformado a partir de la valoración realizada por los sujetos participantes y que no esté influenciada por su perfil demográfico y social. El objetivo final es la definición de una dimensión de la personalidad de la marca que se sume y complemente las dimensiones de la personalidad de la misma, propuestas en el modelo planteado por Aaker (1997).

Las hipótesis generales del trabajo son las siguientes:

Hipótesis 1: Existen unas características, adjetivos o atributos que definen y describen la personalidad de una marca socialmente responsable.

Hipótesis 2: Las características, adjetivos o atributos que conforman la personalidad de una marca socialmente responsable son independientes de las características de los sujetos que hacen su valoración, es decir, están exentos de la influencia de las variables sociodemográficas de las personas que otorgan a una marca la dimensión de responsabilidad social.

\section{Método}

\subsection{Instrumentos de recogida de información}

Para conocer y describir las características que reflejan una personalidad con una marcada sensibilidad social, se utiliza el Instrumento para la Valoración de los Atributos definidores de la personalidad de marca Socialmente Responsable (VAP-SR) (Mayorga y Añaños, 2018), cuya primera parte se destina al registro de la valoración que los individuos dan a cada uno de los 30 atributos que el instrumento plantea. Estos atributos son el resultado del análisis léxico y semántico de un total de 275 adjetivos relacionados con la responsabilidad social corporativa (Mayorga y Añaños, 2018) y son los siguientes:
- Bondadoso
- Comprometido
- Entusiasta
- Carismático
- Confiable
- Caritativo
- Desinteresado
- Colaborador
- Ecologista
- Comprensivo
- Empático
- Equitativo
- Especial
- Esperanzador
- Generoso 
RLCS, Revista Latina de Comunicación Social, 75, 97-120

[Investigación] DOI: 10.4185/RLCS-2020-1418| ISSN 1138-5820 | Año 2020

- Honesto

- Humanitario

- Incansable

- Integrador

- Modesto
- Noble

- Optimista

- Positivo

- Protector

- Respetuoso
- Responsable

- Sensible

- Servicial

- Sincero

- Solidario

En la primera parte del VAP-SR los sujetos deben responder en una escala de Likert de 5 ítems (donde 1 significa Nada y 5 significa Completamente), en qué medida cada uno de los calificativos identifican la descripción de una persona que tiene un marcado interés social (Mayorga, 2017). La tabla 1 muestra los adjetivos evaluados y las escalas de Likert correspondientes.

Tabla 1. Relación de los adjetivos y opciones de respuesta del VAP-SR.

\begin{tabular}{|l|l|l|l|l|l|}
\hline \multicolumn{1}{|c|}{ Adjetivo } & Nada & Muy Poco & Medianamente & Mucho & Completa mente \\
\hline Sincero/a & & & & & \\
\hline Desinteresado/a & & & & & \\
\hline Humanitario/a & & & & & \\
\hline Caritativo/a & & & & & \\
\hline Protector/a & & & & & \\
\hline Ecologista & & & & & \\
\hline Especial & & & & & \\
\hline Comprometido/a & & & & & \\
\hline Integrador/a & & & & & \\
\hline Entusiasta & & & & & \\
\hline Responsable & & & & & \\
\hline Comprensivo/a & & & & & \\
\hline Generoso/a & & & & & \\
\hline Esperanzador/a & & & & & \\
\hline Bondadoso/a & & & & & \\
\hline Noble & & & & & \\
\hline Respetuoso/a & & & & & \\
\hline Optimista & & & & & \\
\hline Empático/a & & & & & \\
\hline Positivo/a & & & & & \\
\hline Servicial & & & & & \\
\hline Incansable & & & & & \\
\hline Modesto/a & & & & & \\
\hline Confiable & & & & & \\
\hline Honesto/a & & & & & \\
\hline Solidario/a & & & & & \\
\hline Carismático/a & & & & & \\
\hline Sensible & & & & & \\
\hline Equitativo/a & & & & & \\
\hline Colaborador/a & & & & & \\
\hline & & & & & \\
\hline
\end{tabular}

Fuente: elaboración propia.

La segunda parte del cuestionario tiene como finalidad adquirir información sobre el perfil demográfico de los sujetos en relación a su edad, género, nivel académico, país de origen, actividad laboral y nivel de participación en actividades de tipo social.

\subsection{Población y muestra}

La selección de los individuos participantes se llevó a cabo a través de un tipo de muestreo no probabilístico con informantes estratégicos en cadena, tipo bola de nieve exponencial. Se elige este tipo de muestreo porque la dinámica de distribución del $V A P-S R$ se hace a través de las redes sociales que se soportan en plataformas digitales virtuales. Dicho muestreo se comporta de forma óptima en este tipo de 
situaciones, ya que un individuo hace de multiplicador logrando llegar a un mayor número de personas en un menor tiempo.

El cálculo del tamaño de la muestra se determina a partir de la fórmula:

$$
n=\left(z_{\alpha / 2} \frac{\sigma}{d}\right)^{2}+1=\left(\frac{z_{\alpha / 2}}{k}\right)^{2}+1
$$

En base a las recomendaciones de Hair, Tatham, Anderson, Black y Babin (2005), considerando que el número de variables es de 36 , asumiendo una confianza del $95 \%$ para la estimación de cada uno de los promedios y considerando también el error máximo admisible como la décima parte de la desviación estándar, el tamaño requerido de la muestra de sujetos participantes es de 385 sujetos. La muestra final de sujetos participantes en la investigación es de 408 sujetos, por lo que se cumple el criterio de representación de la misma. El criterio de selección de los sujetos se establece en base a las características planteadas por Lam (2005), es decir, ser mayor de 16 años y tener un nivel de escolaridad obligatorio.

\subsection{Variables y Tipo de Diseño}

En base al objeto de estudio, las variables que estructuran el análisis de esta investigación son las siguientes:

- Variables Dependientes: Son las variables que resultan de la valoración de cada uno de los atributos del VAP-RS. Hay pues tantas variables de este tipo como adjetivos considerados en el VAP-SR y son: Bondadoso, Carismático, Caritativo, Colaborador, Comprensivo, Comprometido, Confiable, Desinteresado, Ecologista, Empático, Entusiasta, Equitativo, Especial, Esperanzador, Generoso, Honesto, Humanitario, Incansable, Integrador, Modesto, Noble, Optimista, Positivo, Protector, Respetuoso, Responsable, Sensible, Servicial, Sincero, Solidario.

- Variables Independientes: Son las variables que se recogen en la segunda parte del VAP-SR y corresponden a las características sociodemográficas y sociales de los sujetos participantes: edad, género, nivel académico, país de origen, actividad laboral y participación en actividades de tipo social.

El diseño de la investigación se desarrolla en base a los objetivos de la misma, es decir, al análisis de la valoración que otorgan los individuos a las características que describen una personalidad con una sensibilidad manifiesta hacia los demás. Considerando la clasificación propuesta por Ato, López y Benavente (2013), se utiliza un diseño con una estrategia descriptiva de carácter selectivo y de medidas independientes en el cual todos los sujetos se someten a las mismas condiciones de evaluación.

\subsection{Procedimiento}

La investigación se lleva a cabo a partir de la aplicación de la versión digital del VAP-SR (Mayorga, 2017). Dicho cuestionario se distribuye de forma virtual a través de las redes sociales de los participantes. Se determina el uso de internet como medio para la distribución del formulario, ya que según Llieva et al. (2002) (citado por (Díaz de Rada, 2012)), la utilización de este medio optimiza la distribución y la respuesta de un gran número de cuestionarios con una mayor rapidez y con un menor coste de procedimiento. 
Para conseguir la participación de los sujetos se lleva a cabo el muestreo en cadena tipo bola de nieve exponencial (comentado anteriormente). Para la distribución del formulario, se diseña una estructura conformada por los siguientes agentes: el investigador principal, un grupo de sujetos llamados promotores de primer nivel y un grupo de sujetos llamados multiplicadores. El investigador principal se encarga de determinar y seleccionar a los promotores de primer nivel (con quienes se pone en contacto) y les da las indicaciones para que promuevan el formulario a través de sus redes sociales digitales y demás medios virtuales que consideren importantes; el objetivo de este procedimiento es conseguir el mayor número de respuestas y de sujetos multiplicadores; los sujetos multiplicadores, a su vez, también son agentes de promoción del formulario y así sucesivamente. Durante el procedimiento de distribución del VAP-SR se desarrollan diversas acciones de comunicación y promoción que tienen como objetivo conseguir el mayor número de individuos participantes.

\section{Resultados}

\subsection{Descripción de los sujetos participantes}

Los resultados del análisis de la información obtenida con la aplicación del VAP-SR permiten describir las características de la muestra de sujetos participantes. Así, la mayoría de los sujetos son adultos jóvenes y adultos medios que representan el $60,05 \%$ de la muestra. En cuanto al género de los participantes, la muestra está formada por un $62 \%$ de mujeres, un $34,1 \%$ de hombres y un $3,9 \%$ de sujetos que no suministró información al respecto. Los resultados reflejan una distribución de la formación académica de los sujetos que va desde la educación superior hasta el nivel de doctorado, en el que el 71,8\% de los sujetos tiene un nivel profesional, universitario o de posgrado; el 66,42\% de los participantes presenta una vinculación de dependencia laboral, es decir, se encuentra activo laboralmente.

En cuanto al análisis de los rasgos sociales de los sujetos, la mayoría de ellos (el 73,8\%) están vinculados a algún tipo de acción social. Los resultados específicos obtenidos en relación a esta variable son los siguientes:

El 78,8\% de las personas cuya edad está comprendida entre los 25 y los 50 años manifiestan que han realizado o realizan alguna actividad de tipo social y de ellos, el 78,7\% de las mujeres afirma haber hecho alguna actividad de este tipo. De los sujetos trabajadores, el 81,3\% también respondió positivamente en relación a su vinculación social y de los sujetos empresarios la mayoría (el 88,2\%) afirma haber desarrollado o estar desarrollando alguna actividad de tipo social.

Estas características de los sujetos participantes en el estudio son importantes ya que permiten considerar la validez interna de las respuestas conseguidas, puesto que la mayoría de sujetos conocen de forma experiencial el concepto sobre el cual el VAP-SR evalúa la persona con un marcado componente de responsabilidad social.

\subsection{Resultados del análisis de los atributos que componen la dimensión de la personalidad de marca socialmente responsable}

El análisis del grado de valoración de cada uno de los atributos se cuantifica mediante el empleo de indicadores relativos a los rasgos estadísticos que corresponden a las distribuciones de sus frecuencias: 
la posición, la variabilidad y la forma. Con este fin, se calcula: el promedio aritmético (o media aritmética), la desviación estándar y el coeficiente de asimetría de Fisher. La tabla 2 muestra los resultados de estos indicadores en cada uno de los adjetivos evaluados, ordenados a partir de la magnitud del promedio (media obtenida), pudiéndose observar que el atributo que obtiene una mayor valoración es Humanitario y el que obtiene la valoración inferior es Modesto. Además, los resultados obtenidos a partir del análisis del coeficiente de asimetría de Fisher tienen la misma tendencia que la jerarquía obtenida en la valoración realizada a partir de los resultados obtenidos de la media aritmética o promedio.

Tabla 2. Resultado de los parámetros de distribuciones de los atributos y su jerarquización.

\begin{tabular}{|l|c|c|c|c|}
\cline { 5 - 5 } \multicolumn{1}{c|}{ Adjetivo } & Jerarquia & Promedio & $\begin{array}{c}\text { Desviación } \\
\text { estándar }\end{array}$ & Asimetría \\
\hline Humanitario & 1 & 90,69 & 15,432 & $-3,211$ \\
\hline Solidario & 2 & 88,27 & 20,850 & $-2,604$ \\
\hline Generoso & 3 & 86,73 & 21,274 & $-2,356$ \\
\hline Servicial & 4 & 86,73 & 21,992 & $-2,289$ \\
\hline Colaborador & 5 & 86,14 & 20,451 & $-2,167$ \\
\hline Bondadoso & 6 & 84,78 & 21,608 & $-2,177$ \\
\hline Caritativo & 7 & 81,87 & 23,150 & $-1,646$ \\
\hline Comprometido & 8 & 78,44 & 26,538 & $-1,540$ \\
\hline Sensible & 9 & 76,91 & 27,593 & $-1,387$ \\
\hline Responsable & 10 & 72,44 & 30,512 & $-1,059$ \\
\hline Empático & 11 & 69,60 & 31,746 &,- 887 \\
\hline Noble & 12 & 69,45 & 32,318 &,- 892 \\
\hline Ecologista & 13 & 66,59 & 30,631 &,- 757 \\
\hline Respetuoso & 14 & 66,02 & 33,915 &,- 730 \\
\hline Protector & 15 & 63,41 & 31,977 &,- 584 \\
\hline Especial & 16 & 62,98 & 34,216 &,- 589 \\
\hline Positivo & 17 & 62,57 & 33,863 &,- 568 \\
\hline Equitativo & 18 & 61,84 & 32,912 &,- 504 \\
\hline Esperanzador & 19 & 61,34 & 32,668 &,- 468 \\
\hline Comprensivo & 20 & 60,60 & 34,665 &,- 489 \\
\hline Desinteresado & 21 & 60,32 & 39,946 &,- 551 \\
\hline Carismático & 22 & 59,73 & 34,066 &,- 439 \\
\hline Honesto & 23 & 58,51 & 36,208 &,- 390 \\
\hline Entusiasta & 24 & 57,20 & 34,648 &,- 332 \\
\hline Confiable & 25 & 56,73 & 35,022 &,- 292 \\
\hline Optimista & 26 & 54,85 & 35,407 &,- 227 \\
\hline Integrador & 27 & 53,64 & 34,481 &,- 168 \\
\hline Sincero & 28 & 53,32 & 37,838 &,- 173 \\
\hline Incansable & 29 & 51,32 & 36,440 &,- 100 \\
\hline Modesto & 30 & 39,01 & 36,547 &, 394 \\
\hline
\end{tabular}

Fuente: elaboración propia.

El análisis de la relación entre la valoración de cada uno de los atributos y las características de los sujetos se lleva a cabo mediante la utilización de una prueba de independencia estadística, cuantificada a través del coeficiente de contingencia de Cramer.

La tabla 3 muestra una síntesis de los resultados del análisis de las valoraciones de los 30 adjetivos del VAP-SR (posición, media, dispersión y coeficiente de asimetría) así como también los resultados del análisis de las pruebas de independencia estadística de cada atributo (variables independientes) con cada una de las variables (dependientes) relacionadas con las características demográficas y sociales de los sujetos participantes. La última columna muestra el número de condiciones de exclusión de cada 
adjetivo del constructo de la personalidad marcada por un fuerte componente de responsabilidad social. Las condiciones de exclusión de un atributo son las siguientes: 1) el valor $p$, derivado de la prueba de independencia de la valoración del atributo con cada una de las variables sociodemográficas, debe ser significativo, es decir, inferior a $0,01(\mathrm{p}<0,01) ; 2)$ la media aritmética obtenida en la valoración del atributo debe tener un valor pequeño, concretamente situado en las últimas 5 posiciones de la jerarquización obtenida; 3) la desviación estándar obtenida en la valoración del atributo debe situarse entre las cinco valoraciones más altas de la jerarquización; 4) el valor del coeficiente de asimetría debe ser alto y concretamente debe situarse entre los cinco coeficientes más altos obtenidos en la jerarquización (Mayorga, 2017). 
RLCS, Revista Latina de Comunicación Social, 75, 97-120

[Investigación] DOI: 10.4185/RLCS-2020-1418| ISSN 1138-5820| Año 2020

Tabla 3. Tabla Resumen del resultado del Mecanismo de decisión.

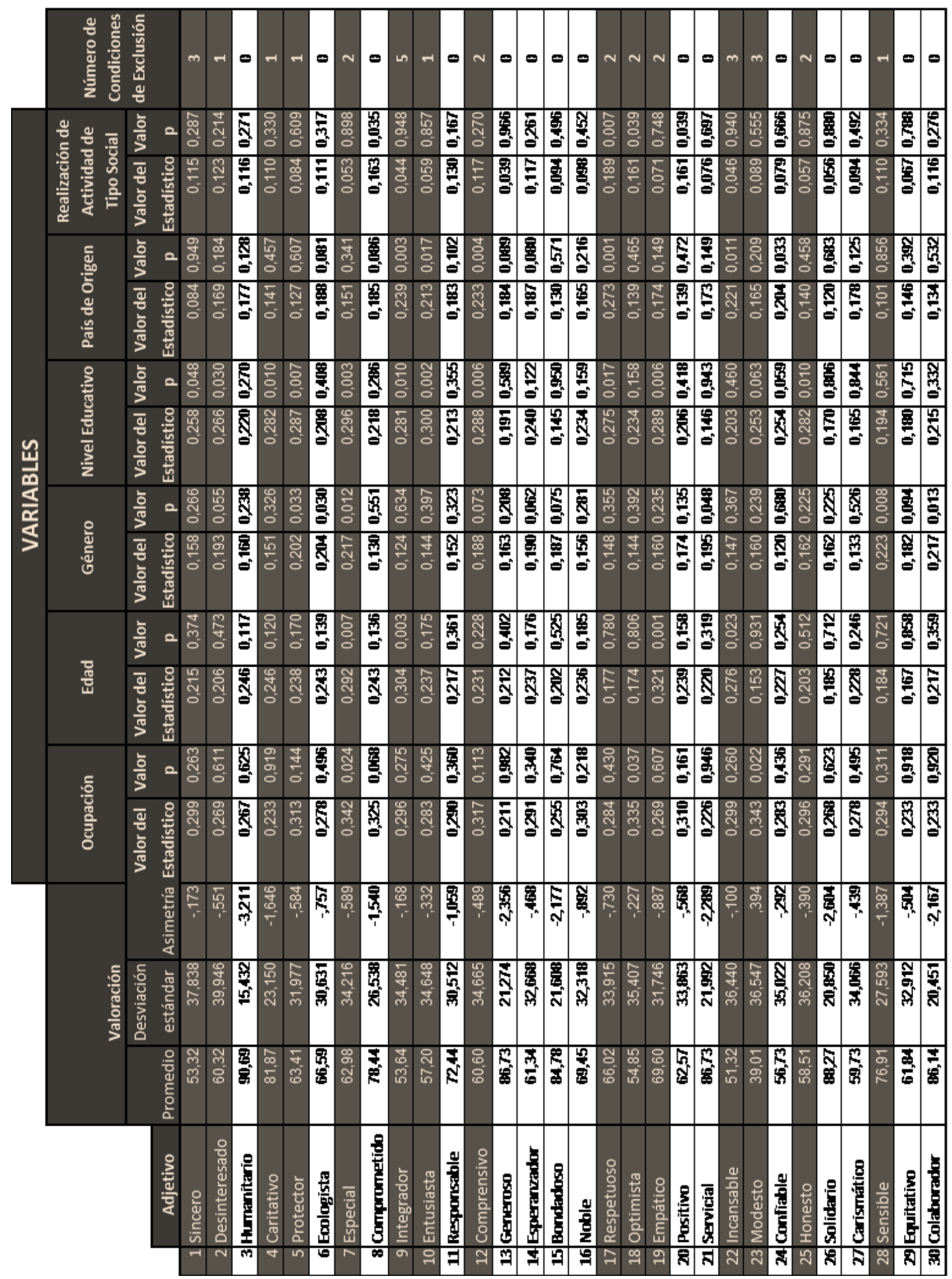

Fuente: elaboración propia. 
En síntesis, los resultados de la tabla 3 muestran que la valoración de los atributos Especial, Integrador y Empático depende estadísticamente $(\mathrm{p}<0,01)$ de la variable edad. En relación al género, los resultados muestran que la intensidad de la dependencia estadística es significativa $(\mathrm{p}<0,01)$ en el atributo Sensible.

En relación a los resultados obtenidos en el análisis de la variable nivel educativo, éstos muestran cómo la valoración de los atributos Caritativo, Protector, Especial, Integrador, Entusiasta, Comprensivo, Empático y Honesto depende de forma estadísticamente significativa $(\mathrm{p}<0,01)$ de esta variable.

Respecto a la ocupación laboral, los resultados indican que esta variable no muestra ninguna relación estadísticamente significativa en la valoración de ningún atributo ( $\mathrm{p}>0,01)$, es decir, la valoración de los atributos es independiente de las condiciones laborales de los sujetos.

En la variable vinculación de los sujetos con actividades de tipo social, los resultados muestran que únicamente existe una relación estadísticamente significativa de esta variable $(\mathrm{p}<0,01)$ en la valoración del atributo Respetuoso.

En base a los resultados obtenidos y a los criterios definidos de inclusión/exclusión de los atributos en la definición de la personalidad con un marcado componente social, se procede a la exclusión de los atributos del proceso de estructuración de la dimensión de responsabilidad social cuando su valoración depende (relación estadísticamente significativa $\mathrm{p}<0,01$ ) de los niveles o categorías de alguna de las variables relacionadas con las características personales de los sujetos, o bien cuando su valoración es exigua, es decir, no pertinente, o cuando la variabilidad extrema de su valoración lo convierte en un rasgo inestable. La Tabla 4 muestra los atributos excluidos y en ella también aparece el número de condiciones de exclusión que obtienen cada uno de ellos.

Tabla 4. Listado de atributos excluidos del proceso de estructuración de la dimensión de la personalidad con el componente de responsabilidad social.

\begin{tabular}{|l|l|}
\cline { 2 - 2 } \multicolumn{1}{c|}{} & \multicolumn{1}{c|}{$\begin{array}{c}\text { Número de } \\
\text { Condiciones } \\
\text { Conivo }\end{array}$} \\
\hline Sincero & \\
\hline Desinteresado & $\mathbf{3}$ \\
\hline Caritativo & $\mathbf{1}$ \\
\hline Protector & $\mathbf{1}$ \\
\hline Especial & $\mathbf{1}$ \\
\hline Integrador & $\mathbf{2}$ \\
\hline Entusiasta & $\mathbf{5}$ \\
\hline Comprensivo & $\mathbf{1}$ \\
\hline Respetuoso & $\mathbf{2}$ \\
\hline Optimista & $\mathbf{2}$ \\
\hline Empático & $\mathbf{2}$ \\
\hline Incansable & $\mathbf{2}$ \\
\hline Modesto & $\mathbf{3}$ \\
\hline Honesto & $\mathbf{3}$ \\
\hline Sensible & $\mathbf{2}$ \\
\hline
\end{tabular}

Fuente: elaboración propia. 
Así pues, de los resultados obtenidos se desprende que los atributos que deben incluirse en la estructuración de la dimensión de la personalidad socialmente responsable son los siguientes:

- Humanitario

- Ecologista

- Comprometido

- Responsable

- Generoso
- Esperanzador

- Bondadoso

- Noble

- Positivo

- Servicial
- Confiable

- Solidario

- Carismático

- Equitativo

- Colaborador

\section{Discusión y conclusiones}

La personalidad de la marca es un concepto clave en la gestión empresarial que se puede utilizar para crear una diferenciación competitiva. Es por ello que en la actualidad, el estudio de la personalidad de la marca ha llevado a distintos autores a estudiar los diversos elementos que fortalecen dicho constructo, proporcionándole no solo una mayor relevancia, sino también formalizándolo y actualizándolo. En este sentido y de acuerdo con Freling et al. (2011) existen una serie de beneficios que se desprenden del hecho de tener una personalidad de marca favorable como la mejora de las actitudes hacia la propia marca y el aumento de las intenciones de compra y de los niveles de confianza y lealtad del consumidor. Así, una marca que esté pensada desde el punto de vista relacional mejorará la capacidad del cliente para interpretar y procesar su información, aumentará la confianza en la decisión de compra y mejorará la calidad de la experiencia del usuario en relación a la marca; en definitiva, la marca ganará valor para el fabricante (Aaker y Álvarez del Blanco, 2014). El estudio de la personalidad de marca es importante pues, para conocer cómo se fraguan los lazos de valor entre las marcas y las audiencias; en el contexto actual es importante que estos lazos se establezcan y se basen a partir de la definición de la marca que contenga una alta sensibilidad social y ambiental.

Hasta hoy el modelo prevalente sobre la personalidad de la marca es el basado en las dimensiones de la personalidad de la marca propuestas por Aaker (1997), en el cual no se detecta explícitamente ningún elemento que permita describir una personalidad de la marca con un componente de responsabilidad social. Son varios los autores que han estudiado la personalidad de la marca desde diferentes puntos de vista e incluso han hecho validaciones del modelo de la autora en diferentes contextos, agregando nuevos elementos e incluso proponiendo modelos diferentes (Delgado, 2014; Geuens, Weijters, y De Wulf, 2009; Goñi et al., 2013; Japutra y Molinillo, 2017; Kim et al., 2013; Ortegon, 2010; Ortíz, 2013; Wang, Wang, Fang, y Jiang, 2018); sin embargo en ninguno de ellos se ha definido la dimensión de la personalidad de la marca socialmente responsable.

Siendo el propósito principal de esta investigación definir una dimensión de tipo socialmente responsable de la personalidad de marca se puede concluir que este trabajo establece un conjunto de características o atributos que contribuyen a la descripción de una personalidad con una marcada tendencia al compromiso social. La aplicación de estos atributos a la gestión de la propia marca constituye una aportación de suma importancia dentro del contexto actual de la sociedad, puesto que, según recogen Sachs y Vilà (2015) en el momento actual la sociedad se encuentra viviendo una era basada en la sostenibilidad. Los resultados de la investigación permiten concluir que existen quince rasgos o atributos que contribuyen en la construcción de una imagen de la marca con una alta sensibilidad social; estos atributos son los siguientes: Humanitario, Ecologista, Comprometido, Responsable, Generoso, Esperanzador, Bondadoso, Noble, Positivo, Servicial, Confiable, Solidario, Carismático, Equitativo y Colaborador. La primera hipótesis planteada en este trabajo, queda pues 
aceptada. Además, el análisis estadístico de la independencia de la valoración de estos atributos de las características demográficas y sociales de los sujetos permite la obtención de argumentos cuantitativos que respaldan también la segunda hipótesis formulada.

Puesto que la valoración de estos rasgos o atributos es independiente de las características demográficas y sociales de los sujetos, se puede considerar que la dimensión socialmente responsable propuesta contiene unos caracteres que, al ser trasferidos a la personalidad de marca, están exentos de la influencia contextual de las audiencias y adicionalmente se puede concluir que tales calificativos o atributos pueden ayudar a los gestores de las marcas a incorporarlos en su definición y en sus estrategias para conseguir así que las marcas sean identificadas, diferenciadas y posicionadas como marcas socialmente responsables.

Los resultados obtenidos en este trabajo son relevantes desde la perspectiva de la gestión empresarial ya que en el actual entorno competitivo marcado por la gestión de la sostenibilidad, la construcción de una de marca fuerte es un elemento central de la estrategia corporativa de cualquier organización. En este contexto y de acuerdo con Manzano et al. (2013), consideramos que la apropiada gestión de un activo intangible como es la marca, de forma que sea difícil de imitar por los competidores, se convierte en una pieza clave sobre la que se sustentará gran parte del éxito de la empresa. Así pues, otra de las contribuciones de este trabajo la forman las aportaciones teóricas relacionadas con la gestión de las marcas con una acentuada sensibilidad social y ambiental, puesto que la incorporación de estos rasgos contribuirá al establecimiento de unas relaciones más duraderas entre el fabricante y su público, ya que la valoración de estos atributos por parte de los consumidores es importante en el proceso de toma de sus decisiones.

Además, de acuerdo con Aldás (2013), consideramos que las iniciativas de responsabilidad social corporativa pueden influir en el valor de marca de las empresas. Para ello su comunicación debe proyectar un comportamiento motivado socialmente y éste debe ser identificado por el consumidor. Los resultados de esta investigación permiten identificar los atributos que pueden conformar una dimensión de la personalidad de marca que denoten una actitud de la marca positiva frente a las necesidades actuales del contexto social. Si además consideramos que un gran número de consumidores hoy en día toman sus decisiones de compra a partir de valoraciones de las aportaciones de la marca a la sostenibilidad, la importancia de estos atributos cobra aún mayor relevancia.

Una de las limitaciones de esta investigación viene dada por el hecho que se ha realizado en un contexto hispano parlante. Por ello consideramos importante su aplicación en poblaciones con rasgos culturales diferentes, para poder generalizar de esta forma en qué medida (o no) estos atributos describen, también en otros contextos, la personalidad de la marca con rasgos socialmente responsables.

\section{Referencias bibliográficas}

Aaker, D. A. y Álvarez del Blanco, R. M. (2014). Las Marcas según Aaker: 20 principios para conseguir el éxito. Empresa Activa (Urano). http://cataleg.uab.cat/record=b1931861 S1*cat

Aaker, J. (1997). Dimensions of Brand Personality. Journal of Marketing Research, 34, 347. https://doi.org/10.2307/3151897 
RLCS, Revista Latina de Comunicación Social, 75, 97-120

[Investigación] DOI: 10.4185/RLCS-2020-1418| ISSN 1138-5820 | Año 2020

Ahmad, A. \& Thyagaraj, K. S. (2017). An empirical comparison of two brand personality scales: Evidence from India. Journal of Retailing and Consumer Services, 36 (January), 86-92. https://doi.org/10.1016/j.jretconser.2017.01.006

AMA. (2015). Common Language in Marketing. http://marketing-dictionary.org/ama

Ato, M., López, J. y Benavente, A. (2013). Un sistema de clasificación de los diseños de investigación en psicología Introducción Un marco conceptual para la investigación empírica en psicología. Anales de Psicología, 29, 1038-1059. http://www.redalyc.org/pdf/167/16728244043.pdf

Becker-Olsen, K. L., Cudmore, B. A. \& Hill, R. P. (2006). The impact of perceived corporate social responsibility on consumer behavior. Journal of Business Research, 59(1), 46-53. https://doi.org/10.1016/j.jbusres.2005.01.001

Casanoves, J. (2017). Fundamentos de Branding. Claves para construir una marca poderosa. Profit Editorial. www.profiteditorial.com

CEPAL (2018). Agenda 2030 y los Objetivos de Desarrollo Sostenible, Una oportunidad para América Latina y el Caribe. https://doi.org/10.1017/CBO9781107415324.004

Chu, S.-C. \& Sung, Y. (2011). Brand personality dimensions in China. Journal of Marketing Communications, 17(3), 163-181. https://doi.org/10.1080/13527260903387931

Cochran, P. L. (2007). The evolution of corporate social responsibility. Business Horizons, 50(6), 449454. https://doi.org/10.1016/j.bushor.2007.06.004

Cuevas Moreno, R. (2011). Las dimensiones de la responsabilidad social de las empresas: una guía de lectura para su estudio, Revista Facultad de Ciencias Económicas: Investigación y Reflexión, XIX(1), 7-26. http://dialnet.unirioja.es/servlet/articulo?codigo=4239475

Delgado, C. (2014). La Personalidad de Marca de las Organizaciones No Lucrativas: Medición y Análisis de la Personalidad de Marca de las ONL Internacionales en el Perú. Universidad Autónoma de Barcelona.

Díaz de Rada, V. (2012). Ventajas e inconvenientes de la encuesta por internet. Papers, 97(1), 193-223. https://doi.org/10.5565/rev/papers/v97n1.71

Du, S., Bhattacharya, C. B. \& Sen, S. (2007). Reaping relational rewards from corporate social responsibility: The role of competitive positioning. International Journal of Research in Marketing, 24(3), 224-241. https://doi.org/10.1016/j.ijresmar.2007.01.001

Dueñas Ocampo, S., Perdomo-Ortiz, J. y Villa Castaño, L. E. (2014). El concepto de consumo socialmente responsable y su medición. Una revisión de la literatura. Estudios Gerenciales, 30(132), 287-300. https://doi.org/10.1016/j.estger.2014.01.022 
RLCS, Revista Latina de Comunicación Social, 75, 97-120

[Investigación] DOI: 10.4185/RLCS-2020-1418| ISSN 1138-5820 | Año 2020

Faircloth, J. B., Capella., L. M. \& Alford, B. L. (2001). The effect of brand attitude and brand imge on brand equity.pdf. Journal of Marketing Theory \& Practice, 9(3).

Freling, T. H., Crosno, J. L. \& Henard, D. H. (2011). Brand personality appeal: Conceptualization and empirical validation. Journal of the Academy of Marketing Science, 39(3), 392-406. https://doi.org/10.1007/s11747-010-0208-3

Geuens, M., Weijters, B. \& De Wulf, K. (2009). A new measure of brand personality. International Journal of Research in Marketing, 26(2), 97-107. https://doi.org/10.1016/j.ijresmar.2008.12.002

Goñi, N., Torres, E. y Aguilera, S. (2013). Dimensiones de la personalidad de la marca en México. Revista de Ciencias Sociales, XIX(2), 213-225.

Hair, J., Tatham, R., Anderson, R., Black, W. \& Babin, B. (2005). Multivariate data analysis. Pearson Prentice Hall. http://cataleg.uab.cat/record=b1694281 S1*cat

Hildebrand, D., Demotta, Y., Sen, S. \& Valenzuela, A. (2017). Consumer responses to corporate social responsibility (CSR) contribution type. Journal of Consumer Research, 44(4), 738-758. https://doi.org/10.1093/jcr/ucx063

Hoeffler, S. \& Keller, K. L. (2002). Building Brand Equity Through Corporate Societal Marketing. Journal of Public Policy \& Marketing, 21(1), 78-89. https://doi.org/10.1509/ippm.21.1.78.17600

ISO (2018). Brand evaluation - Principles and fundamentals.

https://www.iso.org/obp/ui/\#iso:std:iso:20671:dis:ed-1:v1:en

Japutra, A. \& Molinillo, S. (2017). Responsible and active brand personality: On the relationships with brand experience and key relationship constructs. Journal of Business Research, (August), 1-8. https://doi.org/10.1016/j.jbusres.2017.08.027

Kahn, M. E. (2015). A Review of The Age of Sustainable Development by Jeffrey Sachs. Journal of Economic Literature, 53(3), 654-666. https://doi.org/10.1257/jel.53.3.654

Keller, K. L. \& Lehman, D. R. (2006). Brands and Branding: Research Findings and Future Priorities. Marketing Science, 25(6), 740-759. https://doi.org/10.1287/mksc.1050.0153

Kim, Y. K., Shim, S. W. \& Dinnie, K. (2013). The Dimensions of Nation Brand Personality: A Study of Nine Countries. Corporate Reputation Review, 16(1), 34-47. https://doi.org/10.1057/crr.2012.22

Kotler, P., Kartajaya, H. \& Setiawan, I. (2010). Marketing 3.0. Ediciones de la U, Ed.

Lam, R. (2005). Metodología para la confección de un proyecto de investigación. Revista Cubana de Hematología, Inmunología y Hemoterapia, 21.

http://scielo.sld.cu/scielo.php?script=sci_arttext\&pid=S0864-02892005000200007\&lng=es\&nrm=iso 
RLCS, Revista Latina de Comunicación Social, 75, 97-120

[Investigación] DOI: 10.4185/RLCS-2020-1418| ISSN 1138-5820 | Año 2020

Madden, T. J., Roth, M. S. \& Dillon, W. R. (2012). Global Product Quality and Corporate Social Responsibility Perceptions: A Cross-National Study of Halo Effects. Journal of International Marketing, 20(1), 42-57. https://doi.org/10.1509/jim.11.0016

Madrigal, R. \& Boush, D. M. (2008). Social responsibility as a unique dimension of brand personality and consumers' willingness to reward. Psychology and Marketing, 25(6), 538-564. https://doi.org/10.1002/mar.20224

Manzano, J. A., Simó, L. A. y Pérez, R. C. (2013). La responsabilidad social como creadora de valor de marca: El efecto moderador de la atribución de objetivos. Revista Europea de Dirección y Economía de la Empresa, 22(1), 21-28. https://doi.org/10.1016/j.redee.2011.09.001

Mayorga, J. (2017). Personalidad de la marca socialmente responsable. Universidad Autónoma de Barcelona. http://hdl.handle.net/10803/456576

Mayorga, J. y Añaños, E. (2018). Identificación de atributos comunicacionales para la construcción de una imagen empresarial socialmente responsable en la Sociedad del Conocimiento. En Asociación Española de Investigación de la Comunicación (Ed.), 'Comunicación y Conocimiento' - Libro de comunicaciones. Actas del VI Congreso Internacional de la AE-IC (pp. 2482-2500). Salamanca: Asociación Española de Investigación de la Comunicación. Recuperado a partir de http://aeic.org/final/Libro de Comunicaciones del VI congreso AE-IC.pdf

Newholm, T. \& Shaw, D. (2007). Studying the ethical consumer: a review of research. Journal of Consumer Behaviour, 6(5), 253-270. https://doi.org/10.1002/cb.225

Orteg, L. (2010). Panorama actual de los métodos de medición de marcas y valor de marca desde la orientación al consumidor, Poliantea, 6(11), 171-188.

Ortíz, R. (2013). El Efecto De La Imagen Del País De Origen Y La Personalidad De Marca En La Intención De Compra: Un Estudio Transcultural. UNIVERSIDAD DEL TURABO.

http://ut.suagm.edu/sites/default/files/uploads/Centro-Estudios-

Doctorales/Tesis Doctorales/2014/ROrtiz.pdf

RAE (2016). Diccionario de la lengua española. $\underline{\text { www.rae.es }}$

Rivera, J. J., Bigne, E. y Curras-Pérez, R. (2016). Efectos de la responsabilidad social corporativa sobre la satisfacción del consumidor con la marca. Spanish Journal of Marketing - ESIC, 20(2), 104-114. https://doi.org/10.1016/j.sjme.2016.06.002

Sachs, J. y Vilà, R. (2015). Introducción al desarrollo sostenible. En La Era del Desarrollo Sostenible, Nuestro futuro está en juego: incorporemos el desarrollo sostenible a la agenda política mundial (p. 606). https://www.planetadelibros.com/libros_contenido_extra/31/30978_La_era_del_desarrollo_sostenible.pdf

Sen, S. \& Bhattacharya, C. B. (2001). Does Doing Good Always Lead to Doing Better? Consumer Reactions to Corporate Social Responsibility. Journal of Marketing Research, 38(2), 225-243. https://doi.org/10.1509/jmkr.38.2.225.18838 
RLCS, Revista Latina de Comunicación Social, 75, 97-120

[Investigación] DOI: 10.4185/RLCS-2020-1418| ISSN 1138-5820 | Año 2020

Wang, X., Wang, X., Fang, X. \& Jiang, Q. (2018). Power distance belief and brand personality evaluations. Journal of Business Research, 84(November 2017), 89-99.

https://doi.org/10.1016/j.jbusres.2017.11.011

Wartick, S. \& Cochran, P. (1985). The evolution of the corporate social performance model. Academy of management review, 10(4), 758-769.

\section{AUTORES:}

\section{Javier Andrés Mayorga Gordillo}

Departamento de Mercadeo. Universidad Autónoma de Occidente. Colombia.

Publicaciones más recientes: - Capitulo de Libro: Comunicación Experiencial, el nuevo rol de la Gestión Estratégica de Comunicación (ISBN-13:978-84-16458-82-0 / D.L.: TF-1149-2017 / DOI (del libro): 10.4185/129) http://www.revistalatinacs.org/17SLCS/2017 libro/016 Mayorga.pdf. - El Factor Humano, el punto de contacto más relevante de la comunicación corporativa en la actualidad - Revista Questiones Publicitarias - UAB: http://www.questionespublicitarias.es/article/view/v1-n21-mayorga. Identificación de atributos comunicacionales para la construcción de una imagen empresarial socialmente responsable en la Sociedad del Conocimiento. Actas del VI Congreso Internacional de la AE-IC, Comunicación y Conocimiento. https://dd.uab.cat/record/191673?ln=ca /ISBN: 978-84-0903393-5 http://ae-ic.org/final/Libro\%20de\%20Comunicaciones\%20del\%20VI\%20congreso $\% 20 \mathrm{AE}-$ IC.pdf. Premios: - Primer Premio. Degustaciones - Ciclo de Eventos Jóvenes. Asociación de Organizadores de Fiestas, Reuniones Empresariales y Proveedores de la República Argentina, Buenos Aires. Abril 17 de 2007. - Proyecto ganador. Semana de proyectos jóvenes de investigación y creación. Universidad de Palermo. Buenos Aires, Argentina. Primer cuatrimestre de 2007.

jmayorga@uao.edu.co

Orcid ID: https://orcid.org/0000-0003-3094-7951

\section{Elena Añaños Carrasco}

Departament de Publicitat, Relacions Públiques i Comunicació Audiovisual. Universitat Autònoma de Barcelona. España.

Publicaciones más recientes: - Article: Añaños, E. (2018). El efecto del aroma en la intención de compra de un paquete vacacional. Disertaciones. Anuario electrónico de estudios en Comunicación Social. 11. p. 150 - 168. - Book chapter: Mayorga, J. \& Añaños, E. (2018). Identificación de atributos comunicacionales para la construcción de una imagen empresarial socialmente responsable en la Sociedad del Conocimiento. VV.AA. Comunicación y Conocimiento. - ed. Salamanca. Asociación Española de Investigación de la Comunicación; p. 2482 - 2500. - Article: Astals, A. \& Añaños, E. (2017). Estudi amb la tecnologia de l'eye tracking de l'atenció visual dispensada a la publicitat gràfica no comercial: "Vine a donar sang". Comunicació. Revista de recerca i d'Anàlisi. 34. p. 9 - 32. Article: Jauset-Berrocal, J. A., Martínez, I. \& Añaños, E. (2017). Music learning and education: contributions from neuroscience. Cultura y Educación. 29. p. 833 - 847. - Book chapter: Añaños, E. \& Grau, A. (2017). Efectos de la publicidad basada en la imagen corporal en mujeres con y sin trastorno de la conducta alimentaria (TCA: Estudio comparativo. La omnipresencia de la imagen. Estudios interdisciplinares de la cultura visual. 1 ed. Madrid. Global Knowledge Academics; p. 299 - 316. Book chapter: Rodríguez-Muñoz, L. \& Añaños, E. (2017). Los valores percibidos por los adolescentes en las diferentes categorías de la publicidad de productos de alimentación. La omnipresencia de la 
RLCS, Revista Latina de Comunicación Social, 75, 97-120

[Investigación] DOI: 10.4185/RLCS-2020-1418| ISSN 1138-5820 | Año 2020

imagen. Estudios interdisciplinares de la cultura audiovisual. 1 ed. Madrid. Global Knowledge Academics; p. 317 - 340. - Article: Zhang, B., Añaños, E. \& Zhang, M. (2016). Effectiveness of the Auditory and Visual effects of Chinese e-Magazine on the Graduate Students' Reading Process. Analisi. Quaderns de Comunicació i Cultura. 54. p. 58 - 74. - Article: 2015. Neuriscience and advertising. Attention, Emotion and its relationship to the spots awarded at the international advertising festival in Cannes. Analisi. - Article: Añaños, E. (2015). Adultos mayores y publicidad integrada en TV: atención sin procesamiento. UAB Divulga. Revista digital. http://www.uab.cat/web/detalle-noticia1345680342040.html?noticiaid=1345693354029 - Article: Añaños, E. (2015). EyeTracker Technology in Elderly People: How Integrated Television Content is Paid Attention to and Processed. Comunicar. 45. p. 75 - 83. - Article: Añaños, E. \& Oliver, A. (2015). Atención y atención conjunta a los spots de $T V$. Estudio con la tecnología del eye tracker. Gráfica. 3. p. 103 - 114. - Article: Mas-Manchón, L., Rodríguez-Bravo, A., Montoya-Vilar, N., Morales-Morante, F., Lopes, E., Añaños, E., Peres, R., Martínez, M.E. \& Grau, A. (2015). Valores percibidos en la publicidad de alimentos por parte de jóvenes con y sin trastornos de la conducta alimentaria. Salud Colectiva. 11. p. 423 - 444. - Book chapter: Zhang, B.; Añaños, E. (2014). Study on eye tracking technique: do digital media influence reading comprehension? Javier Sierra Sánchez \& Francisco García García (Coord.). Tecnología y narrativa audiovisual. 1 ed. Madrid. Fragua; p. 525 - 540. 978-84-7074-627-7. - Article: Añaños, E. \& Astals, A. (2013). ¿Imagen o texto? El poder de captar la atención de los elementos gráficos analizado con el Eye Tracker. Gráfica. 1. p. 87 - 98. Proyectos de Investigación activos: - 2017SGR0117. Grup de Recerca en Publicitat i Comunicació Radiofònica (Publiradio). Agència de Gestió d'Ajuts Universitaris $i$ de Recerca. Convocation to fund research groups conducting studies in Catalonia in the different scientific areas in order to promote their activity, scientific, economic and social impact, as well as to foster the international projection of their research. Perona Páez, Juan José. Universitat Autònoma de Barcelona (UAB). 01/01/2017 - 31/12/2020. Premios: - Lòpez Salas, Sandra. Award to the best End of Course Thesis on Gender Perspective of the graduate studies on Advertising and Public Relations of UAB of the school year 2016-17. Redes sociales y marcas deportivas: ¿en fuera de género? 28/11/2017. - Serra Vinardell, Clàudia. Award to the best End of Course Thesis on Gender Perspective of the graduate studies on Advertising and Public Relations of UAB of the school year 2015-16. Obsesión 90-60-90. Relación entre la publicidad y la percepción de la imagen corporal en las adolescentes. 29/11/2016. - Teruel, Anna. Award to the best End of Course Thesis on Gender Perspective of the graduate studies on Audiovisual Communication of UAB of the school year 2015-16 Estereotipos de género en los informativos de televisión: ¿existen unos patrones fijos en los profesionales que los presentan? 29/11/2016. - Añaños, E. \& Valli, A. (2012). FINALIST FOR IAB Europe's Research Awards, CATEGORY AD EFFECTIVENESS.TV Integrated Advertising: Conscious and unconscious processing and effectiveness in young and senior targets. 25/04/2012. elena.ananos@uab.cat

Orcid ID: http://orcid.org/0000-0002-9893-6848 